\title{
Análisis lexicométrico aplicado al estudio de las concepciones de aprendizaje
}

\section{Análise lexicométrica aplicada ao estudo das concepções de aprendizagem}

\section{Lexicometric analysis applied for studying conceptions of learning}

\author{
Walter Terrazas $^{1}$
}

\begin{abstract}
RESUMEN
Las concepciones de aprendizaje han sido principalmente investigadas a través de un enfoque denominado fenomenografía. No obstante su aporte a la sistematización teórica de las mismas, recientemente se ha generado un debate en torno a algunas de sus bases metodológicas y conceptuales. Este artículo aborda esta discusión presentando las concepciones de aprendizaje identificadas a través de un enfoque metodológico diferente, el análisis lexicométrico. Entrevistas semiestructuradas, que incluyeron el proceso y resultado del aprendizaje, fueron aplicadas a 29 estudiantes universitarios de primer año y posteriormente analizadas con el apoyo del programa Alceste. Los resultados indican que las concepciones del aprendizaje comprenden el hecho de memorizar, aplicar y comprender contenidos así como la congruencia entre teoría y práctica. Son discutidos los procedimientos de la metodología utilizada, su rol en la identificación de concepciones de aprendizaje y la comparación con resultados de investigaciones previas.
\end{abstract}

Palabras-clave: aprendizaje; estudiantes universitarios; análisis de contenido.

\section{RESUMO}

Concepções de aprendizagem têm sido investigadas principalmente através de uma abordagem chamada fenomenografia. No entanto, a contribuição para

DOI: $10.1590 / 0104-4060.44222$

1 Universidad Católica del Norte. Antofagasta, Antofagasta, Chile. Av. Angamos, no 0610. E-mail:wterraza@ucn.cl 
a sua sistematização teórica tem gerado recentemente um debate acerca de algumas de suas bases metodológicas e conceituais. Este artigo aborda esta discussão, apresentando as concepções de aprendizagem identificadas através de uma abordagem metodológica de análise diferente, a análise lexicométrica. Entrevistas semiestruturadas, que incluíram o processo de aprendizagem e os resultados, foram aplicadas a 29 estudantes universitários do primeiro ano e, posteriormente, analisadas com o apoio do programa Alceste. Os resultados indicam que as concepções de aprendizagem abarcam o ato de memorizar, aplicar e compreender o conteúdo, assim como a coerência entre teoria e prática. São discutidos os procedimentos da metodologia utilizada, o seu papel na identificação de concepções de aprendizagem e a comparação com resultados de pesquisas anteriores.

Palavras-chave: aprendizagem; estudantes universitários; análise de conteúdo.

\begin{abstract}
Learning conceptions have been mainly investigated through an approach called phenomenography. Although this approach has made contributions to the theoretical systematization of such conceptions, a debate about some of its methodological and conceptual bases has recently been opened. This article addresses this discussion by introducing a different methodological approach to the conceptions of learning: the lexicometric analysis. Data was collected by applying semi-structured interviews, which included the learning process and the results, to 29 freshmen and subsequently analyzed with the aid of the Alceste software. Results show that the conceptions of learning include memorizing, applying and understanding content as well as the congruence between theory and practice. The procedures of the methodology used, their role in identifying conceptions of learning and the comparison with the results of previous research are discussed.
\end{abstract}

Keywords: learning; university students; content analysis.

\title{
Introducción
}

Las concepciones de aprendizaje, definidas como las diversas maneras de percibir y comprender este fenómeno, han llegado a constituir una línea de investigación central para la comprensión del proceso educativo. (LIMA; DUARTE, 2010). Su importancia reside en la asociación que ellas tienen con la manera 
de enfocar y abordar el trabajo académico. (COLLIER-REED; INGERMAN, 2013; PAAKKARI; TYNJÄLÄ; KANNAS, 2011).

Una revisión de la literatura muestra que una parte significativa de investigaciones en este campo ha sido conducida a partir de un enfoque teórico y metodológico denominado fenomenografía. Ésta se constituye inicialmente a partir de las bases empíricas que aportaron los estudios de un grupo de investigadores de Göteborg en los años 70. Es posteriormente que se desarrolla como enfoque de investigación, definiendo sus fundamentos en torno a tres dimensiones: epistemológica, metodológica y conceptual. (ÄKERLIND, 2012).

La dimensión epistemológica alude al modo en que se construye el objeto de investigación, destacando el posicionamiento en una perspectiva de segundo orden. Desde esta óptica, la fenomenografía asume como foco de estudio no un fenómeno en sí mismo, sino la manera en que es experimentado por una persona. (MARTON; BOOTH, 1997).

La dimensión metodológica, cuyo énfasis es la información cualitativa, implica un proceso inductivo de elaboración de categorías de significado. La descripción canónica del análisis subraya la focalización en los datos como totalidad y el carácter iterativo entre éstos e interpretación. (MARTON; BOOTH, 1997). A diferencia de otros enfoques cualitativos que buscan identificar la diversidad de categorías que emergen, la fenomenografía se enfoca en los aspectos que son críticos para diferenciarlas. El resultado concreto del análisis corresponde a un número más limitado de categorías que comprenden diferentes significados y que están ligadas lógicamente formando un conjunto jerárquicamente estructurado denominado espacio de resultado. (ÄKERLIND, 2012).

La dimensión conceptual refiere al conjunto de significados atribuidos al fenómeno en estudio y constituye la unidad de análisis de la fenomenografía. (COLLIER-REED; INGERMAN, 2013). Un trabajo precursor en esta línea lo desarrolló Säljö (1979), el que más tarde fue complementado por Marton, Dall'Alba y Beaty (1993), quienes elaboraron una tipología que distingue seis concepciones, a saber, aprendizaje como: 1) acumulación de conocimientos; 2) memorización y reproducción de información; 3) aplicación de conocimientos; 4) comprensión de contenidos; 5) desarrollo de nuevas perspectivas explicativas; 6) proceso de reconstrucción personal.

Estas concepciones han sido encontradas en algunas investigaciones posteriores llevadas a cabo en variadas muestras. (BOULTON-LEWIS et al., 2004; CANO; CARDELLE-ELAWAR, 2004; PURDIE; HATTIE; DOUGLAS, 1996). Estos resultados han llevado a proponer que las concepciones de aprendizaje estarían constituidas por algunos elementos propios de una situación educativa, los que le darían un cierto carácter universal, no obstante los matices y énfasis que pueden ser aportados desde cada contexto cultural. (LIMA; DUARTE, 
2010; PAAKKARI; TYNJÄLÄ; KANNAS, 2011). Asimismo, categorías complementarias han surgido de otras investigaciones, tales como procesamiento de la información, aprendizaje experiencial (TYNJÄLÄ, 1997) y desarrollo personal, entre otras. (PURDIE; HATTIE; DOUGLAS, 1996).

Además de constituir una tipología de concepciones de aprendizaje, las investigaciones bajo el enfoque fenomenográfico han establecido algunas afirmaciones teóricas relativas a su naturaleza. En primer lugar, estas concepciones forman una jerarquía de complejidad creciente donde las tres primeras serían más simples, circunscritas a una situación académica y centradas en la reproducción de conocimiento, mientras que las tres últimas serían más complejas, trascenderían el contexto académico y estarían centradas en una construcción de conocimiento. (ÄKERLIND, 2012; COLLIER-REED; INGERMAN, 2013). En segundo lugar y asociado al postulado de la complejidad creciente, las concepciones de aprendizaje siguen un patrón evolutivo. Aquellas consideradas complejas son más frecuentes en personas con mayor edad y nivel educativo. (BOULTON-LEWIS; WILLS; LEWIS, 2001; CANO; CARDELLE-ELAWAR, 2004; MARTON; DALL'ALBA; BEATY, 1993).

Las implicancias prácticas de estas afirmaciones, en especial la asunción sobre el nivel de complejidad con que los estudiantes abordarían las tareas académicas según su concepción de aprendizaje, han constituido fuentes importantes de discusión en los últimos años. En particular, algunos autores dirigen la crítica a dos aspectos que van en directa relación con el rol del investigador. Por un lado, la universalidad de la tipología, ya que podría responder al énfasis en los marcos interpretativos de estudios previos por sobre la información del contexto de los datos. (ASHWORTH; LUCAS, 2000; PAAKKARI; TYNJÄLÄ; KANNAS, 2011). Por otro lado, la afirmación acerca de la estructuración jerárquica de las concepciones, pues no se deriva directamente de los datos empíricos y respondería más bien a una interpretación de los investigadores. (ÄKERLIND, 2012; ASHWORTH; LUCAS, 2000).

Estos cuestionamientos han reabierto un antiguo debate abordado inicialmente desde ciertas sugerencias acerca de la manera en que el investigador puede integrar sus marcos interpretativos con los datos empíricos. (RICHARDSON, 1999). Más recientemente, este foco ha dado paso al de algunas consideraciones acerca de la calidad de la investigación fenomenográfica. (ÄKERLIND, 2012; COLLIER-REED; INGERMAN, 2013; KHAN, 2014; SIN, 2010). El resultado ha sido la elaboración de criterios que permiten juzgar la coherencia global de una investigación postulando dos tipos de validez. Por una parte, la validez pragmática que refiere a la medida en la cual los resultados de la investigación contribuyen a comprender mejor el tema en cuestión. Por otra parte, la validez comunicativa o juicio acerca de la plausibilidad de los resultados. 
Sin embargo, estos criterios dejan la validez sujeta al juicio externo. La investigación fenomenográfica no logra, desde su estructura interna, dar cuenta suficiente acerca de los fundamentos que permiten responder a los cuestionamientos sobre la universalidad de la tipología y su estructura jerárquica. Nosotros planteamos que un enfoque metodológico alternativo como el análisis lexicométrico puede ofrecer una vía alternativa a la comprensión de estas interrogantes, pues permite hacer más explícito la relación entre metodología, marcos interpretativos y resultados.

En este artículo vamos a reportar las concepciones de aprendizaje de un grupo de estudiantes universitarios a partir de la aplicación de una metodología alternativa, el análisis lexicométrico. Este enfoque, basado en la aplicación de la estadística a las particularidades de datos textuales, permite analizar e identificar núcleos de significado. (LEBART; SALEM, 1994). Es apoyándose en este procesamiento estadístico (realizado independientemente de un marco conceptual cualquiera) que el investigador puede, en un segundo momento, elaborar el significado e interpretar el material textual. Se aplicó así sistemáticamente el análisis lexicométrico a través del programa computacional Alceste con el objetivo de identificar categorías descriptivas de concepciones de aprendizaje, su relación con aquellas encontradas en investigaciones previas, así como su vinculación con el enfoque metodológico utilizado.

\section{Metodología}

\section{Participantes}

El procedimiento de selección se basó en el criterio de variación máxima (PATTON, 2002), considerando dos dimensiones: la distinción entre disciplinas duras y blandas (BIGLAN, 1973) y el sexo de las personas. Así, los participantes fueron 29 estudiantes universitarios de primer año, seleccionados al azar a partir de la base de datos de alumnos inscritos en la universidad que es el contexto de la investigación. Su promedio de edad fue de 19 años y provenían de dos carreras, 16 pertenecían a licenciatura en comunicación ( 6 de sexo femenino y 10 de sexo masculino) y 13 a licenciatura en física ( 9 de sexo femenino y 4 de sexo masculino). La constitución final del grupo en estudio estuvo sujeta al carácter voluntario de la participación. 


\section{Recolección de información}

Siguiendo la tradición fenomenográfica, el método de recolección de información incluyó entrevistas semiestructuradas. Basándose en una revisión de las investigaciones en el área, dos dimensiones fueron abordadas en este proceso:

a) Definición del aprendizaje, que incluye preguntas relacionadas con las características generales del aprendizaje. (BOULTON-LEWIS; WILLS; LEWIS, 2001; BOULTON-LEWIS et al., 2004; CANO; CARDELLE-ELAWAR, 2004; MARTON; DALL'ALBA; BEATY, 1993; PURDIE; HATTIE; DOUGLAS, 1996);

b) Logro de aprendizaje que incluye preguntas relativas a su definición e indicadores de logro de aprendizaje. (CANO; CARDELLE-ELAWAR, 2004; PURDIE; HATTIE; DOUGLAS, 1996).

La construcción de la guía de entrevista consideró estas dos dimensiones y se desarrolló a partir de tres tipos de preguntas siguiendo lo señalado por Flick (1998). Primero, preguntas abiertas cuya finalidad es situar el contexto del tema tratar. Por ejemplo, ¿De qué manera podría establecerse que el alumno ha logrado un aprendizaje? Segundo, preguntas de verificación obtenidas de las dimensiones conceptuales identificadas en investigaciones previas sobre el tema y cuyo objetivo es profundizar en el mismo ¿Podrías nombrar algunos indicadores que permiten establecer que se ha logrado un aprendizaje?, ilustra este tipo de interrogación. Tercero, preguntas de confrontación cuyo objetivo es llevar al entrevistado a reflexionar sobre sus propias respuestas y, eventualmente, aportar información suplementaria. Por ejemplo, ¿Se podría decir que los indicadores mencionados son suficientes para dar cuenta de un aprendizaje logrado?

\section{Procedimiento de análisis}

Las entrevistas fueron transcritas literalmente, con la sola excepción de algunas expresiones que constituyen deformaciones del lenguaje formal con la finalidad de facilitar el reconocimiento por parte del programa de análisis. Por ejemplo, la palabra estai, parte de la jerga cotidiana fue reemplazada por la forma estás (segunda persona singular del verbo estar).

La categorización de respuestas se realizó a partir de un análisis lexicométrico operado por el programa Alceste. Nociones de la lingüística, tales como la distinción entre enunciado y enunciación y los principios del análisis factorial de correspondencia han constituido las bases para definir un concepto que guía el análisis: la identificación de los denominados mundos lexicales. De acuerdo a esta noción, la esfera representacional se organiza en torno a núcleos de significado que se materializan en el discurso a través del vocabulario movilizado en 
los enunciados. De esta manera, el método lexicométrico Alceste analizará las similitudes y diferencias de vocabulario entre los diversos enunciados de manera a constituir categorías de éstos, cada una refiriendo a un núcleo de significado o mundo lexical. (REINERT, 1999).

A nivel metodológico, se realiza un examen de la distribución de las palabras que componen un discurso y se identifican las coocurrencias lexicales estadísticamente significativas para constituir categorías de enunciados. Por coocurrencia debe entenderse un grupo de palabras utilizadas conjuntamente en el discurso y que, bajo la hipótesis de los mundos lexicales, provienen de un mismo núcleo representacional.

Para el análisis, el texto es transformado a un modo estándar llevando todas las palabras a una forma simple, por ejemplo, convirtiendo todos los plurales a su forma singular y las conjugaciones a su forma en infinitivo. Esta transformación, apoyada en un diccionario y un algoritmo que permite identificar palabras que tienen una misma raíz, busca enriquecer las relaciones estadísticas del análisis, concentrándose en su significación esencial más que en sus formas. (DE ALBA, 2004). Por otra parte, el texto es dividido en unidades de contexto elemental, una operacionalización de los enunciados, y que son definidas a partir de la puntuación natural del texto así como de un número aproximado de 10 a 12 palabras principales (sustantivos, adjetivos y verbos). Estas unidades son, a su vez, reagrupadas en unidades más amplias, llamadas unidades de contexto que serán la base para el análisis.

Una vez preparado, el texto es modelizado por una tabla lexical que pone en relación las unidades de contexto en filas y las palabras en columnas. Finalmente, una clasificación descendente jerárquica, que divide sucesivamente de manera binaria, agrupa estas unidades de contexto elemental en categorías. Dado que la división del texto condiciona la constitución de éstas, se realizan dos clasificaciones descendentes jerárquicas sucesivas sobre unidades de contexto de tamaño ligeramente diferente comparándolas a través de un test chi cuadrado. Se restringe así el número de categorías a las palabras presentes en ambas clasificaciones.

Los resultados de estos procedimientos, que operan automáticamente según los algoritmos estadísticos, comprenden tres principales salidas a partir de las cuales se puede ir configurando el análisis a la luz de la investigación específica. Primero, la clasificación descendente jerárquica que muestra las relaciones de proximidad entre las categorías. Segundo, un registro del vocabulario representativo de cada una de éstas en la forma de árbol, denominada clasificación ascendente jerárquica. Tercero, las concordancias o listado de segmentos del corpus donde aparece cada una de las palabras. 


\section{Resultados}

La Figura 1 muestra las cuatro categorías identificadas a partir de la clasificación descendente jerárquica con su vocabulario respectivo.

FIGURA 1 - CLASIFICACIÓN DESCENDENTE JERÁRQUICA

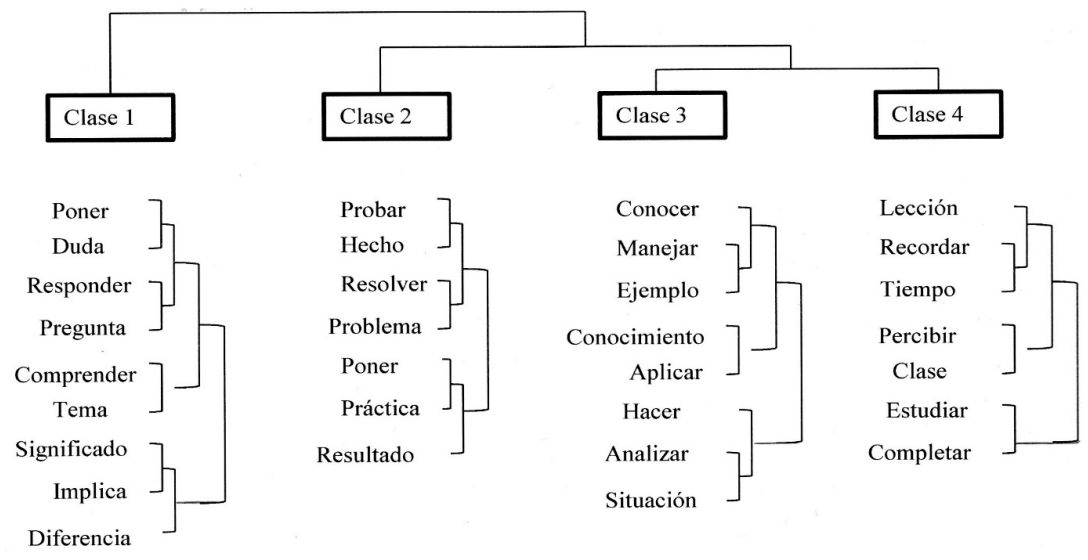

FUENTE: El autor (2016).

El primer nivel de clasificación muestra que la categoría 1 es aquella que se desmarca de todas las demás. El examen de su vocabulario señala que la noción central a partir de la cual se organiza es la de comprensión (comprender-tema) y cuestionamiento (poner-duda).

El segundo nivel de clasificación distingue la categoría 2 de las otras que restan. El examen de su vocabulario muestra que la noción central a partir de la que se organiza es la de prueba empírica (probar-hecho, poner-práctica, resultado).

El tercer nivel de clasificación distingue entre las categorías 3 y 4 . El examen del vocabulario de cada una revela que se distinguen por el hecho que la categoría 3 pone el acento en la aplicación (aplicar-conocimiento, manejar-ejercicios), mientras que la 4 enfatiza la memorización (recordar-lección).

Esta primera caracterización permite identificar la noción central en torno a la cual se articulan las categorías. El contenido de cada una será ahora descrito apelando a las concordancias, es decir, examinando el vocabulario en los segmentos en los que se manifiesta. 
Categoría 1: comprender un tema

En esta categoría, el énfasis está puesto en la dimensión cognitiva del aprendizaje, particularmente, en los elementos que contextualizan una teoría. Así, el aprendizaje es concebido como la comprensión de los argumentos que avalan una determinada proposición teórica. La siguiente cita ilustra esta noción:

Yo me doy cuenta que aprendí algo cuando yo comprendo, por ejemplo, por qué la fuerza de gravedad es 9.8 ... Es por esto y por esto. Es decir, para mi el aprendizaje está logrado. Yo ya comprendí, yo tengo ya en mi mente algo que propuso un señor. Para mí ya es claro, yo comprendo algo que antes no comprendía. (Entrevista 6).

Asimismo, como puede apreciarse en la siguiente cita, procesos como cuestionar la información permiten especificar ciertos medios que demuestran que la comprensión ha tenido lugar.

Yo aprendo algo si puedo ponerlo en duda. Si no conozco algo difíilmente puedo ponerlo en duda. Del momento que puedo poner algo en duda, significa que comprendo la materia, que la puedo cuestionar. (Entrevista 12).

\section{Categoría 2: Congruencia entre teoría y práctica}

En esta categoría, el acento es puesto en la dimensión práctica del aprendizaje. Desde esta perspectiva, se le concibe como la verificación empírica de la teoría. En otros términos, aprender supone constatar que las proposiciones teóricas funcionen en el dominio práctico. Las dos citas siguientes constituyen ejemplos de esta concepción.

En mi opinión, la teoría no sirve de mucho. La teoría es la teoría y siempre debe ser puesta en práctica. Toda teoría debe probarse en la práctica. De hecho, yo no creo en lo que proponen los científicos si yo u otro llega a los mismos resultados... Es como si alguien afirmara la existencia de un avión que puede volar solo. No, debo verlo para creerlo. Yo debo ver la experiencia o alguien debe probarlo. (Entrevista 8). 
Cuando has estudiado, si se quiere ver si uno ha aprendido, se debe someter los contenidos a prueba. Es fundamental reflexionar sobre el tema y poner a prueba los hechos. (Entrevista 19).

\section{Categoría 3: Aplicación de conocimientos}

En esta categoría, se enfatiza también la dimensión práctica del aprendizaje, pero circunscrita al uso de reglas o principios teóricos para abordar problemas asociados a éstos. Así, se concibe el aprendizaje como aplicación o uso de lo aprendido para resolver situaciones o ejercicios relacionados con un determinado contenido. Como se ve en las siguientes citas, esta concepción es expresada de diferentes maneras:

[...] Porque yo puedo aplicar los conocimientos, yo puedo comentar un tema dado, esto significa que yo he aprendido. Por ejemplo, en fisica, yo puedo resolver un problema porque tengo los conocimientos, el aprendizaje. Es de esta manera que yo puedo demostrar que aprendí algo. (Entrevista 1).

El aprendizaje puede verse cuando uno se enfrenta a un ejercicio y es capaz de resolverlo porque tiene los conocimientos y se conoce las respuestas. (Entrevista 13).

\section{Categoría 4: Memorizar la lección}

En esta categoría, el énfasis está puesto en la dimensión cognitiva del aprendizaje, en particular, en el almacenamiento de información. De esta manera, el aprendizaje es concebido como la memorización o retención de los contenidos, particularmente en el contexto de una clase. La siguiente cita ilustra esta concepción:

Yo sé que aprendí algo si puedo recordarlo. Pero si no puedo recordarlo, significa que realmente no lo aprendí. Esto me ocurre a veces con la historia. Yo sé que la lección fue pasada, pero a veces no la recuerdo. (Entrevista 9). 
Al mismo tiempo, en esta clase, se encuentra la idea de que los contenidos de aprendizaje que deben permanecer en la memoria son objeto de un procesamiento destinado a apropiarse de la información. La cita siguiente traduce esta idea:

Se aprende cuando la idea se hace propia, interna. Por ejemplo, cuando hablas con alguien y puedes darle una explicación de algo porque la idea ya ha sido internalizada. Así puedes transmitirla a otro. (Entrevista 16).

\section{Discusión}

El objetivo de esta investigación fue la descripción de las concepciones de aprendizaje de un grupo de universitarios a partir de un enfoque metodológico alternativo, el análisis lexicométrico. En este plano, se puede notar que, aun cuando no hay una coincidencia exacta con los resultados de investigaciones previas, se encuentran algunas categorías comunes tales como memorizar, aplicar y comprender contenidos. Estos hallazgos apoyan la hipótesis de una cierta transversalidad de las concepciones de aprendizaje en torno a diferentes contextos. (LIMA; DUARTE, 2010; PAAKKARI; TYNJÄLÄ; KANNAS, 2011).

Una categoría que se deriva de esta investigación es la que fue denominada congruencia entre teoría y práctica. Aun cuando aparece con un significado que la distingue de las otras, puede observarse también que representa un matiz de mayor sofisticación con respecto a la precedente. En efecto, se puede postular que constituye también una forma de aplicación, pero descrita en términos de mayor complejidad, ya que no se trata de resolver problemas o responder preguntas similares a las aprendidas previamente, como en el caso de la categoría aplicación de conocimientos. Se trata más bien de un proceso donde la dimensión práctica del aprendizaje se visualiza desde un nivel de mayor abstracción que es el de poner en relación dos planos diferentes del conocimiento: el teórico y el empírico.

$\mathrm{Al}$ igual que en la tipología de Marton, la categoría comprender un tema forma parte de un género diferente a las otras, pues es la que en el análisis aparece como aquella que se desmarca con respecto a las demás y, particularmente, de aquella relativa a memorizar la lección. Este hallazgo apoya parcialmente la hipótesis de la estructura en la que se manifiestan las concepciones de aprendizaje, ya que sugiere una mayor oposición entre las categorías relacionadas con 
memorizar y comprender. (ÄKERLIND, 2012; COLLIER-REED; INGERMAN, 2013). Sin embargo, la distinción es sólo de significado, ya que no se encuentran antecedentes suficientes que avalen el postulado de la complejidad creciente de esta estructura. Es decir, las categorías identificadas en esta investigación no traducen la distinción entre concepciones orientadas a la reproducción y concepciones orientadas a la elaboración de significados. Efectivamente, incluso en la concepción memorizar se puede encontrar ideas que expresan una cierta elaboración de la información y el rol activo del sujeto que aprende cuando hace suyas las ideas de un curso.

Por otra parte, las dos últimas categorías de la tipología de Marton relacionadas con el desarrollo de nuevas perspectivas y con un proceso de reconstrucción personal no se manifiestan en esta investigación. Este resultado es consistente con estudios previos que postulan que se trata de concepciones más complejas y cuya manifestación es más frecuente en la medida en que los sujetos tienen niveles evolutivos y educativos más elevados. (BOULTON-LEWIS; WILLS; LEWIS, 2001; CANO; CARDELLE-ELAWAR, 2004; MARTON; DALL'ALBA; BEATY, 1993).

En relación con la exploración de una metodología alternativa, se puede constatar una congruencia a nivel conceptual entre las concepciones del aprendizaje identificadas por medio del análisis lexicométrico y aquellas realizadas por investigaciones previas basadas en la fenomenografía. Efectivamente, los dos enfoques permiten especificar las concepciones en términos de diversos significados atribuidos al aprendizaje, organizados bajo la forma de categorías descriptivas.

Sin embargo, se puede observar una diferencia en la manera en que las categorías son organizadas. En el caso del enfoque fenomenográfico, éstas son definidas a partir de una idea principal. Por el contrario, en el marco del enfoque utilizado en la presente investigación, cada una de ellas corresponde a un contexto que puede agrupar más de una idea representativa de un cierto significado atribuido al aprendizaje. Puede apreciarse, por ejemplo, en la categoría comprender un tema, constituida no sólo por la idea de comprensión, sino también por la de cómo el hecho de poner en duda constituye otra faceta de la misma.

Asimismo, la información de salida del procesamiento llevado a cabo por el programa utilizado, particularmente, la clasificación descendente jerárquica, permite al investigador formular algunas hipótesis acerca de la relación entre las categorías. Así, bajo la premisa de los mundos lexicales, se puede postular que algunas de ellas derivan de núcleos representacionales más próximos, por ejemplo, las categorías aplicación de conocimientos y congruencia entre teoría y práctica. Por el contrario, otras derivarían de núcleos más distantes, por ejemplo, memorizar la lección y comprender un tema. 
Por otra parte, ciertas implicaciones tanto teóricas como metodológicas pueden ser obtenidas de esta investigación. A nivel teórico, sus resultados permiten avalar parcialmente la validez de la tipología propuesta en estudios previos, encontrándose tres de las seis categorías. Estas pueden ser comprendidas como un marco referencial incluyendo esferas de significados atribuidos al aprendizaje cuya descripción debe ser afinada en función de los acentos y matices aportados por cada grupo en estudio. Existe también apoyo para la hipótesis de una estructura que, bajo las premisas de la metodología aplicada en esta investigación, refiere a las similitudes y diferencias entre categorías según provengan o no de un mismo hipotético mundo lexical.

En el plano metodológico, el procedimiento de análisis de datos utilizado es pertinente para la exploración de concepciones de aprendizaje dado la congruencia con la conceptualización presentada por investigaciones previas. Además, este enfoque tiene la ventaja de hacer más explícito el proceso subyacente a la construcción de categorías que realiza el investigador, pues se basa en procedimientos estadísticos que sugieren una clasificación y que son independientes del marco interpretativo de éste. Como señalado previamente, la clasificación descendente jerárquica muestra la relación entre las categorías. Asimismo, la clasificación ascendente jerárquica pone en evidencia las asociaciones entre las palabras que pertenecen a una misma categoría, lo que permite identificar la manera en que el vocabulario se articula en torno a los significados. Del mismo modo, el recurso a las concordancias, es decir, el examen de cada palabra en su contexto de enunciación favorece una descripción del contenido que permite un mayor balance entre aquello que los participantes quisieron expresar y el marco de referencia teórico del investigador, aspectos centrales de un enfoque interpretativo.

Finalmente, no obstante que esta investigación ha permitido mostrar la potencialidad del enfoque metodológico utilizado, la riqueza de la información que se puede obtener está ligada a la extensión del corpus de investigación, asociado a su vez, al tamaño de la muestra. Asimismo, los participantes en esta investigación comprenden dos subgrupos que representan contextos disciplinarios ampliamente diferentes. Estas dos condiciones son las que abren la perspectiva para investigaciones futuras. Por una parte, la aplicación de la metodología en corpus más grandes y, por otra, el examen de similitudes y diferencias en dos contextos disciplinarios diferentes, a saber, el de ciencias básicas y el de ciencias sociales. 


\section{REFERENCIAS}

ÄKERLIND, G. S. Variation and commonality in phenomenographic research methods. Higher Education Research \& Development, v. 31, n. 1, p. 115-127, 2012.

ASHWORTH, P.; LUCAS, U. Achieving empathy and engagement: a practical approach to the design, conduct and reporting of phenomenographic research. Studies in Higher Education, v. 25, p. 295-308, 2000.

BIGLAN, A. The characteristics of subject matter in different academic areas. Journal of applied psychology, v. 57, n. 3, p. 195-203, 1973.

BOULTON-LEWIS, G. M.; MARTON, F.; LEWIS, D. C.; WILLS, L. A. A longitudinal study of learning for a group of indigenous australian university students: dissonant conceptions and strategies. Higher Education, v. 41, p. 97-112, 2004.

BOULTON-LEWIS, G. M.; WILLS, L. A.; LEWIS, D. C. Changes in conception of learning for indigenous australian university students. British Journal of Educational Psychology, v. 71, p. 327-341, 2001.

CANO, F.; CARDELLE-ELAWAR, M. An integrated analysis of secondary school students' conceptions and beliefs about learning. European Journal of Psychology of Education, v. 19, n. 2, p. 167-187, 2004.

COLLIER-REED, B.; INGERMAN, A. Phenomenography: From Critical Aspects to Knowledge Claim. In: TIGHT, M.; HUISMAN, J. Theory and Method in Higher Education Research. Göteborg: Emerald Group Publishing Limited, 2013. p. 243-260.

DE ALBA, M. El método Alceste y su aplicación al estudio de las representaciones sociales del espacio urbano: El caso de la ciudad de México. Papers on Social Representations, v. 13, n. 1, p. 1-20, 2004.

FLICK, U. An introduction to qualitative research. London: Sage publications, 1998.

KHAN, S. H. Phenomenography: A qualitative research method in Bangladesh. International Journal on New Trends in Education and Their Implications, v. 5, n. 2, p. 34-43, 2014.

LEBART, L.; SALEM, A. Analyse statistique des données textuelles: questions ouvertes et lexicométrie. Paris: Dunod, 1994.

LIMA, G.; DUARTE, A. Concepções de aprendizagem em estudantes universitários brasileiros. Psicologia USP, v. 27, n. 4, p. 875-898, 2010.

MARTON, F.; BOOTH, S. Learning and Awareness. New York: Routledge, 1997.

MARTON, F.; DALL'ALBA, G.; BEATY, E. Conceptions of learning. International Journal of Educational Research, v. 19, p. 277-300, 1993. 
PAAKKARI, L. P.; TYNJÄLÄ, P.; KANNAS, L. Critical aspects of student teachers' conceptions of learning. Learning and Instruction, v. 21, n. 6, p. 705-714, 2011.

PATTON, M. Q. Qualitative research \& evaluation methods. London: Sage publications, 2002.

PURDIE, N.; HATTIE, J.; DOUGLAS, G. Student conceptions of learning and their use of self-regulated learning strategies. A cross-cultural comparaison. Journal of Educational Psychology, v. 88, n. 1, p. 87-100, 1996.

REINERT, M. Les “mondes lexicaux" et leur "logique” à travers l'analyse statistique de divers corpus. Lexicometrica, v. 0, p. 1-18, 1999.

RICHARDSON, J. T. E. The concepts and methods of phenomenographic research. Review of Educational Research, v. 69, n. 1, p. 53-82, 1999.

SÄLJÖ, R. Qualitative differences in learning as a function of the learner's conception of the task. Göteborg: Acta Universitatis Gothoburgensis, 1979.

SIN, S. Considerations of Quality in Phenomenographic Research. International Journal of Qualitative Methods, v. 9, p. 305-319, 2010.

TYNJÄLÄ, P. Developing education student's conceptions of the learning process in different learning environments. Learning and Instruction, v. 7, n. 3, p. 277-292, 1997.

Texto recibido en 04 de diciembre de 2015. Texto aprobado en 22 de mayo de 2016. 
